\title{
DELAYED LOGISTIC POPULATION MODELS REVISITED
}

\author{
EDUARDO LIZ \\ Dedicated to Professor Jaume Llibre on the occasion of his 60th birthday
}

\begin{abstract}
We discuss the global dynamics of some logistic models governed by delaydifferential equations. We focus on models of exploited populations, and study the changes in the dynamics as the harvesting effort is increased. We get new results and highlight the link among different logistic equations usually employed in population models.
\end{abstract}

2010 Mathematics Subject Classification: 34K18, 34K20, 92D25.

Key words: Logistic models, delay-differential equations, population dynamics, onedimensional maps, stability, bifurcation, periodic solutions.

\section{Introduction}

Perhaps the best known ordinary differential equation is the Verhulst (logistic) equation

$$
x^{\prime}(t)=r x(t)\left(1-\frac{x(t)}{K}\right),
$$

where $r$ and $K$ are positive constants. Although this equation has been employed in many disciplines, it was originally designed to study the growth of populations with an intrinsic growth rate $r$ and a saturation level $K$ (for historical notes on this equation, see $[\mathbf{2 4 ,} \mathbf{3 5}]$ ). It is this framework of population dynamics the one we are interested in.

Throughout this paper we shall assume that $K=1$. This simplification can be done by a simple change of variables, and it does not affect the dynamics of (1.1).

We will assume that part of the population is removed at a rate proportional to the population size, so we get a different form of the Verhulst 
equation

$$
x^{\prime}(t)=-\delta x(t)+r x(t)(1-x(t))
$$

with $\delta>0$.

In a recent paper, Geritz and Kisdi [14] argued that (1.2) is a form of the logistic equation in which the parameters are more meaningful: if (1.2) is used to model the growth of a population with competition for the available sites, then $\delta$ and $r$ are the per capita death and birth rates, respectively.

We shall refer to the destruction rate $\delta$ as the harvesting effort. Actually, equation (1.2) can be used to model a strategy of harvesting based on constant effort, that is, the catch is proportional to the population size $[4,8]$ (see also $[6,42]$ ). A similar strategy is sometimes used to control plagues or nuisance species using pesticides.

As it has been emphasized in [14], "the effect of changing model parameters is of prime interest to gain insight into the dynamical properties of the system (bifurcation analysis), or to plan interventions concerning natural or exploited populations (management)". In this paper, we analyze the response of the system to an increasing harvesting effort, choosing the culling rate $\delta$ as the bifurcation parameter. We notice that $\delta$ can be regarded as an external control parameter, because managers can make decisions on the harvesting effort.

It is easy to check that if $\delta<r$ then the only equilibria of (1.2) are $x=0$ and $x=1-\delta / r$. The latest one is asymptotically stable, and all solutions $x(t)$ of $(1.2)$ with initial condition $x_{0}>0$ converge to it as $t \rightarrow \infty$. We will denote this positive equilibrium by $K_{\delta}$, to emphasize that $\delta$ is the bifurcation parameter. If $\delta \geq r$, then $x=0$ is the only equilibrium of (1.1) and attracts all solutions. This means that the population is driven to extinction if the harvesting effort is greater than the growth rate $r$. In summary, the main consequences of harvesting are a reduction on the population size as $\delta$ is increased, and extinction if $\delta \geq r$ (what is called overharvesting).

Equation (1.2) cannot explain some observations in laboratory populations; for example, the size of the population cannot oscillate. For this reason, many modifications of Verhulst equation have been proposed. One of the usual ingredients to make the logistic equation more realistic is the consideration of time lags (fecundity rates, necessary time for the resources to recover, etc). In this paper, we revisit the best known delayed logistic models in the context of exploited populations; since they exhibit a rich dynamics, the effects of harvesting are more than merely a reduction of the equilibrium size. 


\section{The discrete logistic model}

A first attempt to explain oscillations in population size was the use of a discrete analogue of the logistic equation:

$$
x_{n+1}=r x_{n}\left(1-x_{n}\right) .
$$

Although discussed in previous work, the discrete logistic (or quadratic) equation (2.1) was popularized by Robert M. May in the 70's (see, e.g., $[\mathbf{3 6}]$ ). This equation is well-known because it is a paradigm of complex behaviour in simple deterministic mathematical models. Depending on the value of $r$, the dynamics ranges between a globally stable equilibrium and chaos (see, e.g., $[\mathbf{1 1}, \mathbf{4 2}]$ ).

If we introduce a proportional harvesting with constant rate $\gamma \in(0,1)$ in (2.1), we get

$$
x_{n+1}=(1-\gamma) r x_{n}\left(1-x_{n}\right)=(r / \delta) x_{n}\left(1-x_{n}\right),
$$

where we write $\delta=1 /(1-\gamma) \in(1, \infty)$ for the sake of comparison with other models. Notice that the positive equilibrium of $(2.2)$ is $K_{\delta}=$ $1-\delta / r$, the same as in (1.2).

A new feature of this harvesting model is that the unharvested population $(\delta=1)$ can be unstable, and in this case increasing harvesting is stabilizing. See Figure 1.

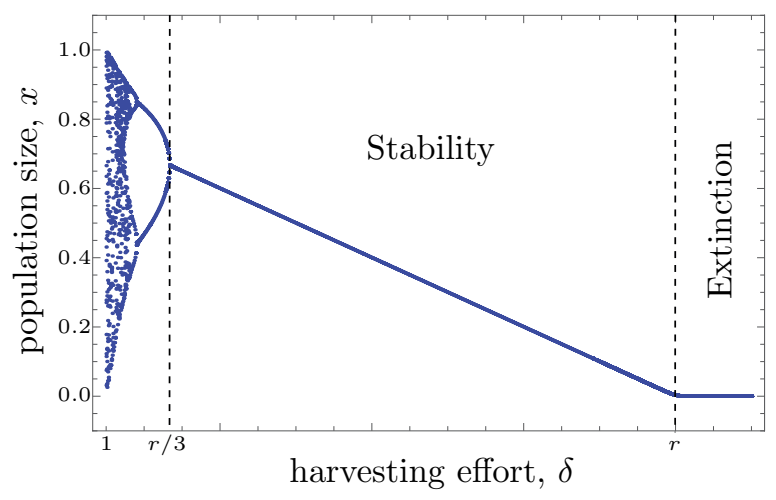

FiguRe 1. Bifurcation diagram of (2.2) for $r=4$ and $\delta \in(1,4]$.

Since the quadratic map is very well studied, we just mention without further details the following effects of harvesting in the discrete model (2.2): 
- As in the Verhulst equation, increasing harvesting reduces population size, and overharvesting leads to extinction (in a tangent bifurcation at $\delta=r$ ).

- Increasing harvesting stabilizes an unstable population (after a period-halving bifurcation at $\delta=r / 3$ ).

- Increasing harvesting reduces fluctuations in population size (the difference between the maximum and the minimum values of the attractor is a monotone nonincreasing function of $\delta$ ).

\section{The Hutchinson-Wright equation}

In 1948, George Evelyn Hutchinson [22] considered a delay $\tau$ in the self-regulatory mechanisms of a population governed by the Verhulst equation. He observed that the time lag induces oscillations, explaining in this way some natural phenomena in animal populations, as Daphnia (water flea).

Assuming that the saturation level is $K=1$, Hutchinson equation writes

$$
x^{\prime}(t)=r x(t)(1-x(t-\tau)),
$$

where $\tau>0$ is the delay.

Equation (3.1) has been extensively studied, starting with a famous paper of Edward Maitland Wright [54]. An equivalent form of (3.1) is known as the Wright equation, so, like other authors, we will adopt the term Hutchinson-Wright equation for (3.1); see, e.g., [25].

Since our motivation comes from populations dynamics, we only consider nonnegative solutions of (3.1). To be more precise, we introduce the notion of admissible solutions.

Definition 1. We say that a continuous function $\phi:[-\tau, 0] \rightarrow \mathbb{R}$ is an admissible initial condition of $(3.1)$ if $\phi(t) \geq 0$ for all $t \in[-\tau, 0]$ and $\phi(0)>0$. Solutions of (3.1) corresponding to admissible initial conditions are called admissible solutions.

It is easy to prove that to an admissible initial condition $\phi$ corresponds a unique admissible solution $x(t)=x(t, \phi)$ of $(3.1)$, which is bounded, positive, and defined on $[-\tau, \infty)$ (see, e.g., [48, Section 5.5]).

The local stability analysis of the equilibrium $K=1$ of (3.1) goes back to a paper of N. D. Hayes [20]. He rigorously proves that $K$ is asymptotically stable if $r \tau<\pi / 2$, and it is unstable if $r \tau>\pi / 2$. This result is stated without proof in the original Hutchinson's paper $[\mathbf{2 2}$, p. 237]. 
Many features are known on the global dynamics of (3.1); see, for example, Chapters XV and XVI in $[\mathbf{1 2}]$, and the recent surveys $[\mathbf{2 6 , 4 6}$. We recall that when $r \tau=\pi / 2$, a supercritical Hopf bifurcation occurs at $x=1$, in such a way that the positive equilibrium loses its stability, and stable periodic orbits appear for $r \tau>\pi / 2$; for a proof, see [48].

Another important property establishes that a Poincaré-Bendixson Theorem holds $[\mathbf{3 4}, \mathbf{5 3}]$, and therefore complex dynamics is not possible: every orbit is bounded and its $\omega$-limit set is either an equilibrium or a periodic orbit.

We do not know any study about the influence of constant effort harvesting in a population governed by (3.1), that is, for equation

$$
x^{\prime}(t)=r x(t)(1-x(t-\tau))-\delta x(t),
$$

where $\delta \geq 0$.

A simple change of variables $y(t)=r(r-\delta)^{-1} x(t)$ transforms (3.2) into equation

$$
y^{\prime}(t)=(r-\delta) y(t)(1-y(t-\tau))
$$

Thus, we get from our previous discussion that the only positive equilibrium $K_{\delta}=1-\delta / r$ of (3.2) is asymptotically stable if $(r-\delta) \tau<\pi / 2$, and it is unstable if $(r-\delta) \tau>\pi / 2$. This fact means that if the positive equilibrium of the unharvested model (3.1) is unstable, that is, if $r \tau>\pi / 2$, it becomes stable as the effort harvesting reaches the critical value $\delta^{*}=r-\pi /(2 \tau)$. At this value, a Hopf bifurcation occurs. We emphasize that extensive analytic and numerical results suggest that $K_{\delta}$ is actually a global attractor of all positive solutions of (3.2) if $\delta<\delta^{*}$ (this statement is popularly known as the Wright conjecture), and there is a nontrivial periodic solution attracting almost all solutions if $\delta>\delta^{*}$ (this postulation is known as the Jones conjecture); for a further discussion, see $[30]$ and references therein. Numerical simulations suggest that the amplitude of the attracting periodic solution is a nonincreasing function of $\delta$.

In summary, as harvesting increases the positive equilibrium is stabilized and there is a reduction in the average and fluctuations in population size. Again, overharvesting drives the population to extinction after a saddle-node bifurcation occurs at $\delta=r$. We can conclude that the effects of harvesting in the Hutchinson-Wright equation and the discrete logistic map are similar; the main difference is that complex behaviour is not possible in the former one. Figure 2 shows a numerical bifurcation diagram for equation (3.2), taking $\delta$ as the bifurcation parameter. We plot the minimum and the maximum values reached by the periodic solutions. For each value of $\delta$, the minimum and the maximum values 
attained by the attracting periodic solution are plotted in blue. The solid red line represents the average value of the periodic solution, which matches the positive equilibrium when it is asymptotically stable, and remains very close to it when it is unstable.

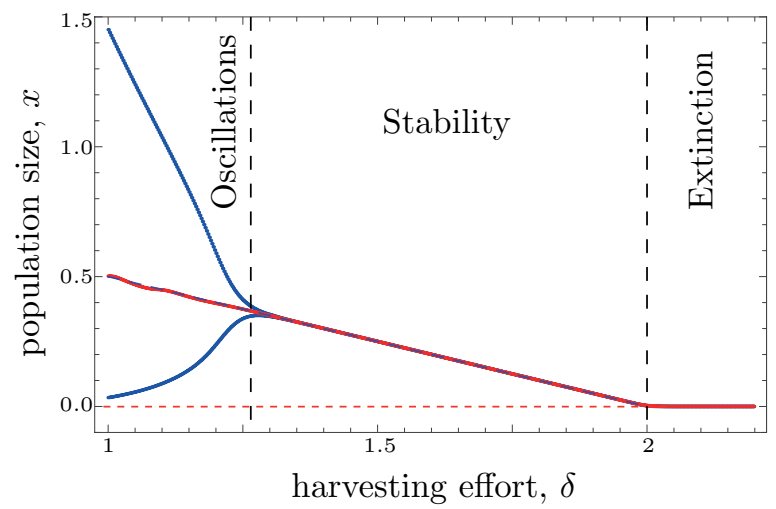

Figure 2. Bifurcation diagram of (3.2) for $r=2, \tau=2$, and $\delta \in[1,2.2]$.

\section{The blowfly logistic equation}

In this section, we consider an equation that is not as well understood as the Hutchinson-Wright equation. To help the reader, we split the section into several subsections, starting with some historical notes.

4.1. History. In 1968, Maynard Smith [40] derived equation

$$
x^{\prime}(t)=-\delta x(t)+r x(t-\tau)(1-x(t-\tau))
$$

to model an age-structured population with two stages: larva and adult. This model assumes that the adult mortality rate $\delta$ is constant and the number of eggs laid by each adult follows the logistic relation. The time lag $\tau$ represents the time taken from egg to adult. In 1976, Charles E. Taylor and Robert R. Sokal [49] investigated the appropriateness of equation (4.1) to describe oscillations of adult numbers in laboratory populations of Musca domestica.

Equation (4.1) is in its own right a Verhulst-type delay differential equation, since for $\tau=0$ it becomes equation (1.2). Notice that (1.2) is also obtained from equation (3.2) with $\tau=0$. From a biological point of view, a drawback of equation (4.1) is that admissible initial conditions 
can produce solutions with unrealistic negative values of the population size for some model parameters.

In 1978, J. F. Perez, C. P. Malta, and F. A. B. Coutinho [44] generalized equation (4.1) to study oscillations of isolated populations of the fly Drosophila stutervantis. They performed the stability analysis of the positive equilibrium in equation

$$
x^{\prime}(t)=-\delta(x(t)) x(t)+b(x(t-\tau)) x(t-\tau) .
$$

Here, the nondecreasing function $\delta(x)$ is the per capita death rate, and the decreasing function $b(x)$ is the per capita birth rate. Hadeler [19] calls (4.2) the blowfly equation. A famous particular case is the Nicholson blowflies equation studied by Gurney et al. [17]; for more examples, see $[\mathbf{6}, \mathbf{4 6}]$.

Perez et al. gave some numerical results for (4.2) with constant $\delta$, and $b(x)=b_{0}-b_{1} x$ if $x \leq b_{0} / b_{1}$, and $b(x)=0$ if $x>b_{0} / b_{1}$. This form of equation (4.2) can be reduced to

$$
x^{\prime}(t)=-\delta x(t)+r x(t-\tau) \max \{1-x(t-\tau), 0\} .
$$

A generalized form of (4.3) was also used by the International Whaling Commission, namely

$$
x^{\prime}(t)=-\delta x(t)+r x(t-\tau) \max \left\{1-x^{z}(t-\tau), 0\right\},
$$

where the positive parameter $z$ measures the severity with which the changes in the population density are registered $[\mathbf{7}, \mathbf{3 7}, \mathbf{4 2}]$. This model was considered by May $[\mathbf{3 7}, \mathbf{3 8}]$ as a continuous version of a difference equation investigated in [7], which is referred to as the Clark equation.

The relationship between equations (4.1) and (4.3) is that they coincide for initial conditions $\phi:[-\tau, 0] \rightarrow[0,1]$ if $\delta>r / 4$. This statement can be easily proved using the invariance principle of Ivanov and Sharkovsky [23, Theorem 2.1].

We concentrate our analysis on equation (4.3), having in mind that its dynamics is valid for (4.1) under certain restrictions on the initial data and the involved parameters. In the following, we refer to (4.3) as the blowfly logistic equation.

As in the previous section, we only consider admissible solutions of (4.3). Since any admissible solution satisfies $x^{\prime}(t) \geq-\delta x(t), x(0)>0$, it follows that $x(t)>0$ for all $t>0$. Moreover, a simple application of the variation of constants formula shows that admissible solutions are defined on $[-\tau, \infty)$ (see, e.g., $[\mathbf{2 6}]$ ). 
4.2. Stability. The mathematical part of the aforementioned papers $[38,44,49]$ is essentially focused on the asymptotic stability of the positive equilibrium. This study involves the linear equation

$$
x^{\prime}(t)=-\alpha x(t)+\beta x(t-\tau),
$$

with $\alpha, \beta \in \mathbb{R}$. A thorough stability analysis of (4.5) can be found in the paper of Hayes [20]; see also [5]. In his famous book [41], John Maynard Smith obtained the stability boundaries for (4.5) in an independent way. This is an important point, because he popularized the linear equation (4.5) among ecologists. Actually, the papers of Taylor-Sokal and Perez-Malta-Coutinho use Smith's approach. May [38] performed his own analysis.

We summarize the stability results for (4.3). If $r<\delta$, then the only equilibrium is $x=0$, and it attracts all positive solutions; if $r>\delta$, then there is a positive equilibrium $K_{\delta}=1-\delta / r . K_{\delta}$ is locally asymptotically stable whenever $p \leq 2$, or $p>2$ and $0<\tau<\tau^{*}$, where

$$
\tau^{*}=\frac{\arccos (1 /(1-p))}{\delta \sqrt{(1-p)^{2}-1}} ; \quad p=\frac{r}{\delta}-1 .
$$

As a limit form of (4.6), we get that the positive equilibrium is asymptotically stable whenever $r \tau>\pi / 2$, regardless the value of $\delta$. See Figure 4.

As far as we know, global stability conditions are not available in the literature for equation (4.3). We include here a result in this direction.

First, we recall two definitions. If $h: I \rightarrow I$ is a continuous map defined on a real interval $I$ and $x^{*}$ is a fixed point of $h$, then we say that $x^{*}$ is a global attractor of $h$ on $I$ if $\lim _{n \rightarrow \infty} h^{n}(x)=x^{*}$ for all $x \in I$, where $h^{n}$ denotes the $n$th iteration of $h$.

We say that the positive equilibrium $K_{\delta}$ of (4.3) is globally asymptotically stable if it is asymptotically stable and $\lim _{t \rightarrow \infty} x(t)=K_{\delta}$ for all admissible solutions $x(t)$ of (4.3).

Theorem 1. Assume that $1<r / \delta<4$, and denote $p=(r / \delta)-1 \in$ $(0,3)$. Then, the positive equilibrium $K_{\delta}=1-\delta / r$ of (4.3) is globally asymptotically stable if either $p \leq 2$ or $p>2$ and

$$
0<\tau<\frac{1}{\delta} \ln \left(\frac{p-1}{p-2}\right) .
$$

Proof: Consider the map

$$
g(x)=\frac{1}{\delta} \max \{r x(1-x), 0\} .
$$


It is clear that $K_{\delta}$ is the unique positive fixed point of $g$, and

$$
\max \{g(x): x>0\}=g(1 / 2)=\frac{r}{4 \delta}<1 .
$$

Thus, $g$ maps $(0,1)$ in $(0,1)$.

We claim that if $x$ is an admissible solution of (4.3), then there exists $T>0$ such that $x(t)<1$ for all $t \geq T$. Assume first that $x(t) \geq 1$ for all $t>0$. Then, $x^{\prime}(t)=-\delta x(t), \forall t>0$, implying that $\lim _{t \rightarrow \infty} x(t)=0$, a contradiction. Therefore, there exists $t_{1}>0$ such that $x\left(t_{1}\right)<1$. We prove by contradiction that $x(t)<1$ on $\left[t_{1}, \infty\right)$. Assume this claim is false; then there is a point $t_{2}>t_{1}$ such that $x\left(t_{2}\right)=1, x(t)<1$ on $\left(t_{1}, t_{2}\right)$. In consequence,

$$
0 \leq x^{\prime}\left(t_{2}\right)=-\delta x\left(t_{2}\right)+\delta g\left(x\left(t_{2}-\tau\right)\right)=\delta\left(-1+g\left(x\left(t_{2}-\tau\right)\right)\right)<0,
$$

a contradiction.

Hence, we can restrict our attention to solutions of (4.3) taking values on $(0,1)$, and therefore Theorem 2.2 in [23] and Corollary 17 in [18] apply to ensure that the equilibrium $K_{\delta}$ of (4.3) is globally asymptotically stable if at least one of the following conditions holds:

(a) $K_{\delta}$ is a global attractor of $g$ on $(0,1)$, or

(b) $K_{\delta}$ is a global attractor on $(0,1)$ of the map

$$
F(x)=e^{-\delta \tau} K_{\delta}+\left(1-e^{-\delta \tau}\right) g(x) .
$$

Since both $g$ and $F$ are quadratic maps, condition (a) holds if $g^{\prime}\left(K_{\delta}\right) \geq$ -1 (which is equivalent to $p \leq 2$ ), and (b) holds if $F^{\prime}\left(K_{\delta}\right) \geq-1$, which is equivalent to (4.7).

In Figure 3, we plot the boundaries of the regions of local and global stability for equation (4.3) in the plane of parameters $(\delta \tau, p)$, where $p=(r / \delta)-1 \in(0,3)$. The horizontal line $p=2$ is an asymptote of both curves. The upper dashed line represents the border of the asymptotic stability region; the solid line corresponds to the global stability condition (4.7); finally, the horizontal dotted line $p=2$ denotes the border of the absolute (delay-independent) stability.

A consequence of Theorem 1 is that, for sufficiently large values of the harvesting effort $\delta(p \leq 2 \Leftrightarrow \delta \geq r / 3)$, all positive solutions of (4.3) converge to the positive equilibrium $K_{\delta}$, regardless the value of the time lag parameter $\tau$. Thus, increasing $\delta$ is stabilizing; this effect becomes clear in Figure 4, where we plot the border of the local stability region given by (4.6) in the plane of parameters $(\delta \tau, r \tau)$. 


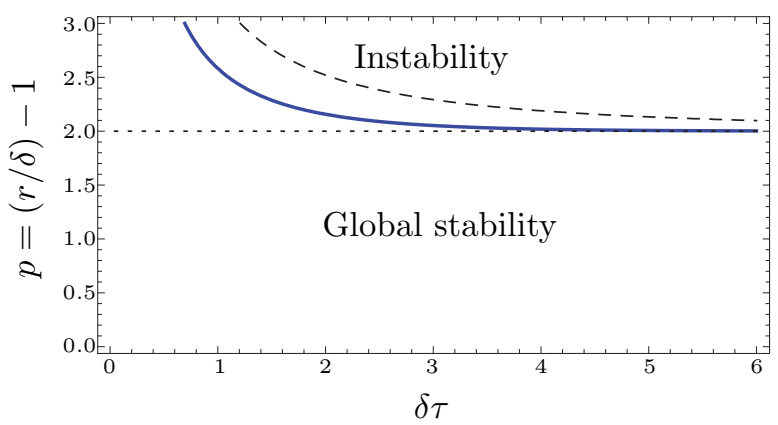

FIGURE 3. Stability diagram for equation (4.3) in the plane of parameters $(\delta \tau, p), p=(r / \delta)-1$.

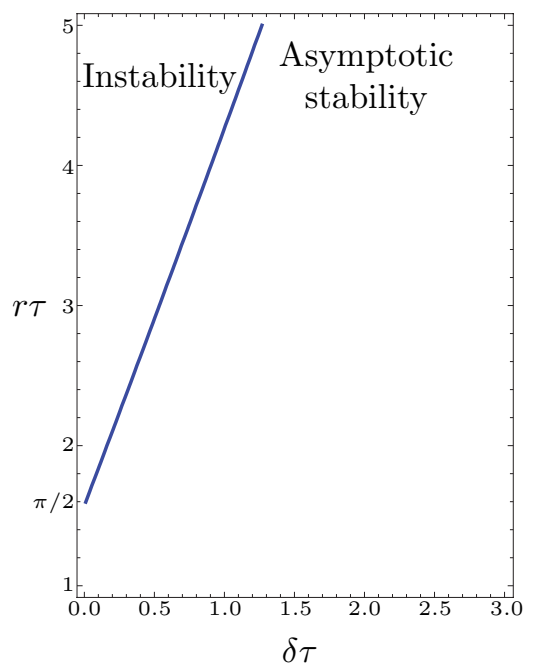

Figure 4. Stability diagram for equation (4.3) in the plane of parameters $(\delta \tau, r \tau)$, showing that increasing $\delta$ is stabilizing.

4.3. Global dynamics. To discuss the global behaviour of the solutions as the parameter $\delta$ is increased, we perform some numerical simulations for (4.3) with $r=2$, and two different values of the time lag: $\tau=2$ and $\tau=10$.

For the smaller value of $\tau$, no complex behaviour is observed: the positive equilibrium seems to be globally attracting when it is asymptotically stable, and there is a simple attracting nontrivial periodic solution when the equilibrium is unstable. 
The bifurcation diagram in Figure 5 is similar to a bubble, but we underline that a stable positive equilibrium cannot be destabilized increasing $\delta$. The phenomenon of bubbling is explained in [27]; for a bubble to exist, the positive equilibrium is first destabilized and then stabilized again in two consecutive Hopf bifurcations as $\delta$ is augmented. Bubbles have been observed for some delay differential equations, including the Nicholson blowflies equation introduced in [17].

In Figure 5 the red solid line represents the mean population size. For small values of $\delta$, there are sustained oscillations, but complex behaviour is not observed. In contrast with the Hutchinson-Wright equation, the amplitude of the periodic solutions is not a monotone decreasing function of $\delta$, and the average value of the solutions can be larger than the positive equilibrium $K_{\delta}$.

Figure 5 points out two phenomena that are not observed in the Hutchinson-Wright equation:

1. Greater harvesting can magnify oscillations in population abundance.

2. Mean population abundance can increase with increasing harvesting.

Both phenomena are of paramount importance in population management. Especially, if the target of increasing mortality is control of plagues, both effects are undesirable, and it is important to determine the range of values of $\delta$ for which they can happen. The first phenomenon has been observed in fishing populations $[\mathbf{2 1}, \mathbf{4 7}]$, and the second one is commonly known as the hydra effect, after the nice review of Abrams [1]. For related results in delay-differential equations, see $[\mathbf{2}, \mathbf{5 0}, \mathbf{5 1}]$.

To further illustrate that both the amplitude ant the average of the attracting periodic solution can increase for small values of $\delta$, we plot the attracting periodic solution of (4.3) for two values of $\delta$ in Figure 6 . The blue horizontal line represents the positive equilibrium $K_{\delta}$. The abscissa corresponds to the time $t$, once the transients have died out, and the ordinate is the population size $x$ at time $t$. These figures illustrate the fact that both the amplitude ant the average of the attracting periodic solution can increase for small values of $\delta$.

For larger values of $\tau$, complex behaviour is observed, and the counterintuitive effects on the amplitude and the average value of the solutions are more notable. We plot the bifurcation diagram of (4.3) for $r=2$, $\tau=10$, and $\delta \in[0,1]$ in Figure 7. The red line represents the average of the solutions, that can increase with $\delta$ for small values of the harvesting effort. 




Figure 5. Bifurcation diagram of (4.3) for $r=2, \tau=2$, and $\delta \in[0,2]$.
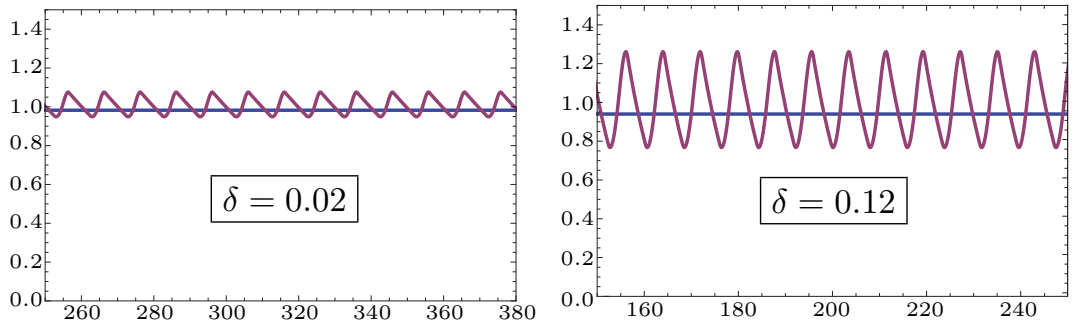

Figure 6. Solutions of (4.3) with $r=2$ and $\tau=2$, and two values of $\delta$.



Figure 7. Bifurcation diagram of (4.3) for $r=2, \tau=$ 10 , and $\delta \in[0,1]$. 
A usual way to show the complexity of the dynamics consists of printing the phase-space portrait of the delay differential equation with $x(t-\tau)$ plotted against $x(t)$. A closed Jordan curve corresponds to a simple limit cycle, with only one maximum in each period interval, and closed curves with self-intersections correspond to more complicated limit cycles (see, e.g., [42, pp. 22-23]). These phase-space portraits are referred to as time delay embeddings in $[\mathbf{1 5}]$. We show some of them for equation (4.3) with $r=2, \tau=10$, and different values of $\delta$ in Figure 8. We observe a period-doubling route to chaos followed by period-halving sequences leading again to simple limit cycles. Similar plots were obtained for the celebrated Mackey-Glass equation [15], but using a different bifurcation parameter. See also the figures obtained by May for equation (4.4) for $\delta=1, r=1$, and $\tau=2$ as the parameter $z$ ranges from $z=3$ to $z=4$.

We draw the reader's attention to the fact that complex behaviour can appear for the range of parameter values for which equations (4.3) and (4.1) do coincide (i.e., for $\delta \geq r / 4$ and initial data on [0,1]). This remark shows that equation (4.1) can exhibit complex dynamics. This fact has not been noticed in previous work; for example Ruan [46, Section 3.2] writes:

"However, unlike the Nicholson model, aperiodic oscillations have not been observed (in the model of Taylor and Sokal)."

On the other hand, seemingly aperiodic solutions are showed in the papers of Perez et al. [44] and May [38]. Actually, Glass and Mackey acknowledge in their review article in Scholarpedia [15] that

"The bifurcations and chaotic dynamics that have been intensively studied and characterized in the Mackey-Glass equation, might have been pursued in the Lasota equation or the Perez-Malta-Coutinho equation but for some small chances of fate."

We end this section with a survey of the effects of harvesting in equation (4.3):

- Overharvesting leads to extinction.

- Increasing harvesting stabilizes an unstable population.

- Increasing harvesting can increase the mean population size (hydra effect).

- Increasing harvesting can magnify fluctuations in population size. 

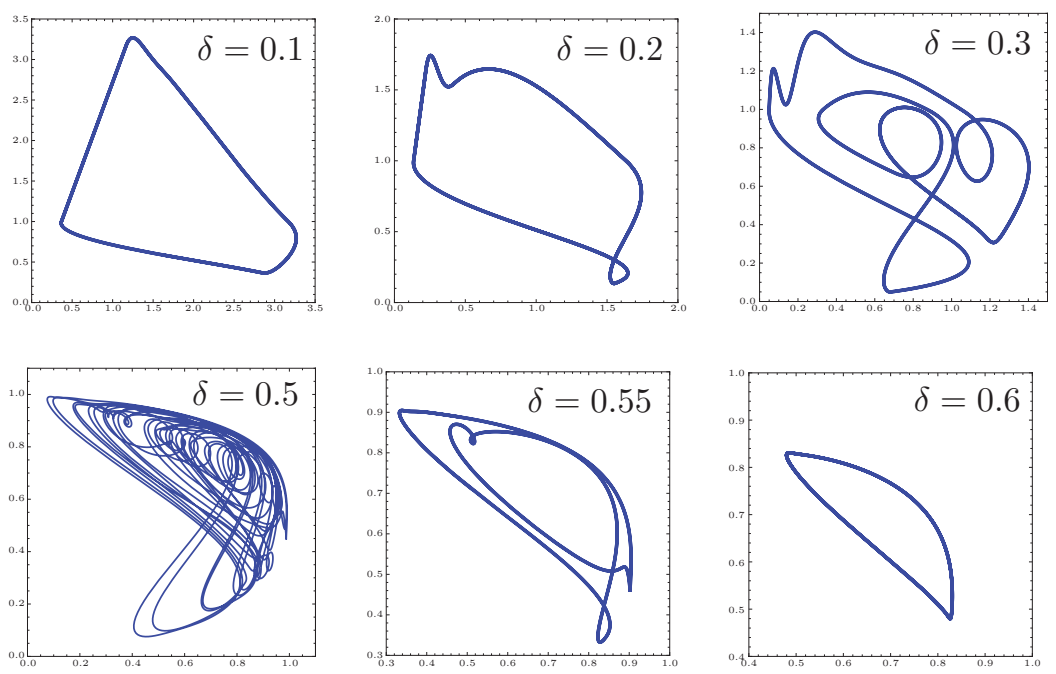

Figure 8. Time delay embedding for equation (4.3) with $r=2, \tau=10$, and different values of $\delta$. The abscissa is $x(t)$ and the ordinate is $x(t-10)$. The corresponding solutions $x(t)$ versus $t$ are plotted in Figure 9 for the three last values of $\delta$.
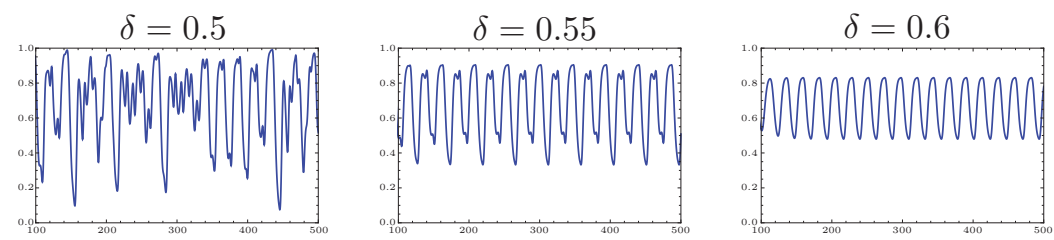

FIgURE 9. Solutions of equation (4.3) with $r=2, \tau=$ 10 , and different values of $\delta$ : a simple periodic solution for $\delta=0.6$, a more complicated periodic solution for $\delta=$ 0.55 , and an apparently aperiodic solution for $\delta=0.5$. The abscissa is $t$ and the ordinate is $x(t)$.

4.4. A mathematical bridge. The rich behaviour exhibited by the solutions of equation (4.3) makes it more close to the discrete logistic equation (2.1). Quoting May [38, p. 272]: 
"Equation (3.1) has the disadvantage that it exhibits either a stable point or simple stable cycles. Neither period doubling effects nor apparent chaos can arise, and its use has unfortunately obscured the similarity that in fact exists between biological extremes of discrete (difference equations) and continuous (differential-delay equations) population growth."

May already considered (2.2) as a "very crude approximation" of (4.3), and he noticed the relationship between the local stability of the positive equilibrium for both models. Here we establish more precisely the link between these two equations.

Equation (4.3) can be transformed via the change of variables $y(s)=$ $x(s \tau)$ into the singularly perturbed equation

$$
\varepsilon y^{\prime}(s)=-y(s)+g(y(s-1)),
$$

where $\varepsilon=1 /(\delta \tau)$, and $g(y)=(r / \delta) \max \{x(1-x), 0\}$. Thus, the limit form of (4.3) as $\varepsilon \rightarrow 0$ is a difference equation with continuous argument, whose dynamics is determined by the dynamics of the one-dimensional map $g$. Notice that, if $\delta$ remains bounded, $\varepsilon$ tends to 0 as $\tau$ tends to infinity. Thus, if $r / \delta \leq 4$ and we restrict the set of initial conditions for (4.3) to

$$
\mathcal{F}=\{\phi:[-\tau, 0] \rightarrow[0,1]: \phi \text { is continuous and } \phi(0)>0\},
$$

we can look at (4.3) as a "bridge" between the ordinary differential equation (1.2) and the discrete logistic model (2.2). The former one is obtained from (1.2) for $\tau=0$, and the latest as a formal limit as $\tau \rightarrow \infty$.

In particular, the delay-independent global stability condition obtained in Theorem 1 is $\delta \geq r / 3$, which is the criterion for the global stability of the positive equilibrium in the discrete logistic equation (2.2).

The proof of Theorem 1 reveals that considering initial values only in the set $\mathcal{F}$ is not a real constraint if $r / \delta \leq 4$ and we are only interested in the long-time behaviour of the solutions of (4.3).

Finally, let us mention that the link between delay-differential equations and one-dimensional maps has been successfully exploited to gain information about the dynamics of the former ones. Pioneering work is due to Mallet-Paret and Nussbaum [32, 33], and Ivanov and Sharkovsky $[\mathbf{2 3}]$. For recent results, see $[\mathbf{2 9}, \mathbf{3 0}, \mathbf{3 1}, \mathbf{4 5}, \mathbf{5 5}]$.

\section{Discussion}

Delayed logistic population models have been criticized many times, but, at the same time, they have been generalized very often, and helped 
to develop the mathematical theory of differential equations and dynamical systems. We refer to the monographs $[\mathbf{1 6}, \mathbf{2 8}, \mathbf{4 8}]$ for further reading on delay-differential equations in the framework of population dynamics.

The recent papers $[\mathbf{3}, \mathbf{4 6}]$ contain interesting discussions on delayed logistic models. Our approach in this paper is different: we focus on the responses of different logistic models to a variation in a model parameter which can be important for management.

As claimed by Arino, Wang and Wolkowicz [3], and Geritz and Kisdi [14], the main drawback of the Hutchinson-Wright equation seems to be that the involved parameters have not a clear interpretation in terms of the behaviour of individuals, that is, (3.1) is not a mechanistic model.

Arino et al. derived an alternative formulation of delayed logistic growth. Their model is a particular case of (4.2), and its main feature is that no solution oscillates. Moreover, its dynamics is very simple: either the only equilibrium is 0 (and it attracts all nonnegative solutions), or there is a positive equilibrium, which attracts all positive solutions.

Geritz and Kisdi also suggest two alternative formulations, and both of them predict a dynamics similar to that found in the model of Arino et al. The form of one of the mechanistic delay equations proposed in [14] is similar to the model introduced in [3]; the other one is different and involves a cross term $x(t) x(t-\tau)$, as in Hutchinson equation. This latter model is quite similar to the delay-differential equation proposed and studied by Cooke $[\mathbf{9}]$ (see also [46, Section 7]) to model the spread of a disease transmitted to the host by an insect vector, assumed to have a large and constant population, and by the host to that vector. Within the vector there is an incubation period $\tau>0$ before the disease agent can infect a host. The model writes

$$
x^{\prime}(t)=-c x(t)+b x(t-\tau)(1-x(t)),
$$

where $b$ and $c$ are positive constants.

The interest of considering models as the blowfly equation studied in Section 4 is that they are able to display a rich behaviour, which explains some phenomena reported in empirical studies. In particular, oscillations and aperiodic behaviour have been often observed in laboratory populations; famous examples are Nicholson's experiments [43] and, more recently, the studies with flour beetles of Cushing et al. [10]. See also $[17,21,22,39,44,47,49]$.

From the mathematical point of view, the role of (4.3) as a formal bridge between the continuous and discrete logistic models is a noteworthy feature. On the one hand, conditions for global attraction in 
the discrete model provide sufficient conditions for the global stability of the positive equilibrium in (4.3) for all values of the delay; on the other hand, the increasing dynamical complexity in (4.3) as the time lag is increased somehow explains the transition between the simple dynamics of the Verhulst equation (1.1) and the chaotic behaviour displayed by the solutions of (2.1) for some parameter ranges. In Figure 10, we sketch the links between the models discussed in the paper.

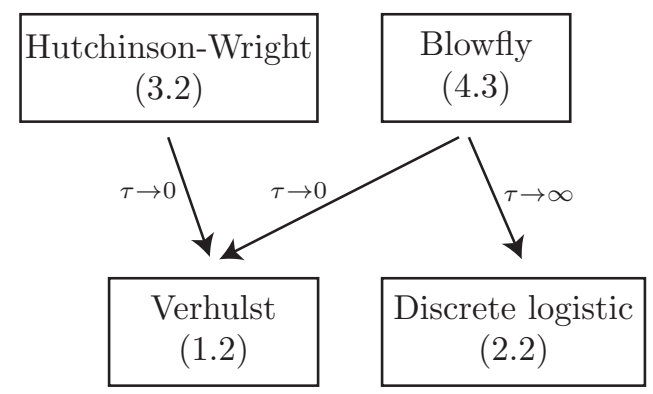

FiguRE 10. Relations among the main equations considered in the paper, emphasizing the role of equation (4.3) as a mathematical bridge between the classical continuous and discrete logistic models.

\section{A more critical discussion}

This last section is devoted to comment some drawbacks of the models considered in this paper that have been raised by one reviewer, and are indeed in order.

First, in regard to the discrete model (2.1) many authors have argued against the name discrete logistic equation because its dynamics is far from the simple behaviour of the solutions of the logistic differential equation (1.1). This point is very well explained in the book of H. R. Thieme [52, Section 9.4]. We refer to the work of Geritz and Kisdi $[\mathbf{1 3}]$ for an interesting discussion on the topic; the authors argue that "the dynamical properties of the discrete and the continuous logistic models are entirely different, and hence the naive discretization of the continuous logistic model is not acceptable as the derivation of the discrete logistic model." However, they used a consumer-resource system with time scale separation to derive the discrete quadratic equation (2.1) for the between-year dynamics of the consumer population when the within-year resource dynamics is given by the continuous logistic equation (1.1). 
Second, it does not seem realistic that a population can survive if the maturation time is too long. This is the reason why we call the link between the ordinary and discrete logistic equations a mathematical bridge. Actually, a more realistic model should take into account a juvenile mortality during the maturation period. This fact has been incorporated to other population models of logistic type; for example in the models of Arino, Wang and Wolkowicz [3], and Geritz and Kisdi [14] mentioned in Section 5, and in Section 3 of [19], where the blowfly equation (4.2) is written in the form

$$
x^{\prime}(t)=-\delta(x(t)) x(t)+e^{-\mu \tau} b_{1}(x(t-\tau)) x(t-\tau) .
$$

The biological interpretation of (6.1) is that adults produce offspring with a birth rate $b_{1}$ but this offspring enters the adult population only after time $\tau$ and with a discount factor $e^{-\mu \tau}$ that accounts for juvenile mortality. Clearly, the limit case of equation (6.1) as $\tau \rightarrow \infty$ does not result in the difference equation (2.1). In fact, an important feature of equation (6.1) is that it predicts extinction of the species for long values of the delay. For example, if $\delta$ is constant and $b_{1}(x)=1-x$, then we get the following variant of (4.1):

$$
x^{\prime}(t)=-\delta x(t)+e^{-\mu \tau} x(t-\tau)(1-x(t-\tau)) .
$$

It is easily seen that equation (6.2) has a positive equilibrium only if $r>\delta$ and $\tau<\tau_{*}=(1 / \mu) \ln (r / \delta)$. It follows from well-known results (see, e.g., [18]) that all admissible solutions of (6.2) converge to zero if $\tau \geq \tau_{*}$.

\section{Acknowledgements}

The author thanks the editors of this special issue for their kind invitation to contribute, and Dr. Gergely Röst for useful discussions. The author also acknowledges the reports of two anonymous reviewers; in particular, the insightful critique of one of them lead to include the final section of the manuscript.

This research was partially supported by the Spanish Government and FEDER, grant MTM2010-14837.

\section{References}

[1] P. A. Abrams, When does greater mortality increase population size? The long history and diverse mechanisms underlying the hydra effect, Ecol. Lett. 12(5) (2009), 462-474. DOI: 10.1111/j.14610248.2009.01282.x. 
[2] P. A. Abrams And C. Quince, The impact of mortality on predator population size and stability in systems with stagestructured prey, Theoret. Population Biol. 68 (2005), 253-266. DOI: $10.1016 / j \cdot \mathrm{tpb} .2005 .05 .004$.

[3] J. Arino, L. Wang, And G. S. K. Wolkowicz, An alternative formulation for a delayed logistic equation, J. Theoret. Biol. 241(1) (2006), 109-119. DOI: 10.1016/j.jtbi.2005.11.007.

[4] J. R. Beddington And R. M. May, Harvesting natural populations in a randomly fluctuating environment, Science 197 (1977), 463-465. DOI: 10.1126/science.197.4302.463.

[5] R. Bellman AND K. L. COOKE, "Differential-difference equations", Academic Press, New York-London, 1963.

[6] F. Brauer And C. CAStillo-ChÁvez, "Mathematical models in population biology and epidemiology", Texts in Applied Mathematics 40, Springer-Verlag, New York, 2001.

[7] C. W. Clark, A delayed-recruitment model of population dynamics, with an application to baleen whale populations, J. Math. Biol. 3(3-4) (1976), 381-391. DOI: 10.1007/BF00275067.

[8] C. W. CLARK, "Mathematical bioeconomics: the optimal management of renewable resources", Pure and Applied Mathematics, Wiley-Interscience [John Wiley \& Sons], New York-London-Sydney, 1976.

[9] K. L. Cooke, Stability analysis for a vector disease model, Conference on Deterministic Differential Equations and Stochastic Processes Models for Biological Systems (San Cristobal, N.M., 1977), Rocky Mountain J. Math. 9(1) (1979), 31-42. DOI: 10.1216/RMJ1979-9-1-31.

[10] J. M. Cushing, R. F. Costantino, B. Dennis, R. A. DeSharnais, And S. M. Henson, "Chaos in Ecology: Experimental Nonlinear Dynamics", Theoretical Ecology Series, Academic Press, New York, 2003.

[11] R. L. DEVANEY, "An introduction to chaotic dynamical systems", Second edition, Addison-Wesley Studies in Nonlinearity, AddisonWesley Publishing Company, Advanced Book Program, Redwood City, CA, 1989.

[12] O. Diekmann, S. A. van Gils, S. M. Verduyn Lunel, and H.O. WAlther, "Delay equations. Functional, complex, and nonlinear analysis", Applied Mathematical Sciences 110, Springer-Verlag, New York, 1995. DOI: 10.1007/978-1-4612-4206-2.

[13] S. A. H. GERITZ AND É. KISDI, On the mechanistic underpinning of discrete-time population models with complex dynamics, J. The- 
oret. Biol. 228(2) (2004), 261-269. DOI: 10.1016/j.jtbi.2004. 01.003.

[14] S. A. H. Geritz AND É. KISDI, Mathematical ecology: why mechanistic models? J. Math. Biol. 65(6-7) (2012), 1411-1415. DOI: 10.1007/s00285-011-0496-3.

[15] L. Glass And M. MACKEy, Mackey-Glass equation, Scholarpedia 5(3) (2009), 6908. DOI: 10.4249/scholarpedia.6908.

[16] K. Gopalsamy, "Stability and oscillations in delay differential equations of population dynamics", Mathematics and its Applications 74, Kluwer Academic Publishers Group, Dordrecht, 1992.

[17] W. S. C. Gurney, S. P. Blythe, and R. M. Nisbet, Nicholson's blowflies revisited, Nature 287 (1980), 17-21. DOI: 10.1038/ $287017 \mathrm{a} 0$.

[18] I. GyöRI AND S. I. Trofimchuk, Global attractivity in $x^{\prime}(t)=$ $-\delta x(t)+p f(x(t-\tau))$, Dynam. Systems Appl. 8(2) (1999), 197-210.

[19] K. P. HADELER, Neutral delay equations from and for population dynamics, in: "The 8th Colloquium on the Qualitative Theory of Differential Equations", Proc. Colloq. Qual. Theory Differ. Equ. 8, Electron. J. Qual. Theory Differ. Equ., Szeged, 2008, 18 pp.

[20] N. D. HAYEs, Roots of the transcendental equation associated with a certain difference-differential equation, J. London Math. Soc. 25 (1950), 226-232. DOI : 10.1112/jlms/s1-25.3.226.

[21] C.-H. Hsieh, C. S. Reiss, J. R. Hunter, J. R. Beddington, R. M. MAY, and G. Sugihara, Fishing elevates variability in the abundance of exploited species, Nature 443 (2006), 859-862. DOI: 10.1038/nature05232.

[22] G. E. Hutchinson, Circular causal systems in ecology, Ann. New York Acad. Sci. 50 (1948), 221-248. DOI: 10.1111/j.1749-6632. 1948.tb39854.x.

[23] A. F. Ivanov And A. N. Sharkovsky, Oscillations in singularly perturbed delay equations, in: "Dynamics reported: expositions in dynamical systems", Dynam. Report. Expositions Dynam. Systems (N.S.) 1, Springer, Berlin, 1992, pp. 164-224.

[24] S. Kingsland, The refractory model: the logistic curve and the history of population ecology, Q. Rev. Biol. 57 (1982), 29-52.

[25] M. Kот, "Elements of mathematical ecology", Cambridge University Press, Cambridge, 2001. DOI : 10.1017/CB09780511608520.

[26] T. Krisztin, Global dynamics of delay differential equations, $P e$ riod. Math. Hungar. 56(1) (2008), 83-95. DOI: 10.1007/s10998008-5083-x. 
[27] T. Krisztin And E. Liz, Bubbles for a class of delay differential equations, Qual. Theory Dyn. Syst. 10(2) (2011), 169-196. DOI: 10.1007/s12346-011-0055-8.

[28] Y. KuANG, "Delay differential equations with applications in population dynamics", Mathematics in Science and Engineering 191, Academic Press, Inc., Boston, MA, 1993.

[29] E. Liz, Un puente entre una ecuación diferencial ordinaria y un sistema dinámico discreto vía ecuaciones diferenciales con retardo, Gac. R. Soc. Mat. Esp. 11(3) (2008), 489-506.

[30] E. Liz And G. Röst, Dichotomy results for delay differential equations with negative Schwarzian derivative, Nonlinear Anal. Real World Appl. 11(3) (2010), 1422-1430. DOI: 10.1016/j.nonrwa. 2009.02.030.

[31] E. LiZ AND G. Röst, Global dynamics in a commodity market model, J. Math. Anal. Appl. 398(2) (2013), 707-714. DOI: 10.1016/ j.jmaa. 2012.09.024.

[32] J. Mallet-Paret and R. D. Nussbaum, Global continuation and asymptotic behaviour for periodic solutions of a differentialdelay equation, Ann. Mat. Pura Appl. (4) 145 (1986), 33-128. DOI: 10.1007/BF01790539.

[33] J. Mallet-Paret and R. D. Nussbaum, A differential-delay equation arising in optics and physiology, SIAM J. Math. Anal. 20(2) (1989), 249-292. DOI: 10.1137/0520019.

[34] J. Mallet-Paret and G. R. Sell, The Poincaré-Bendixson theorem for monotone cyclic feedback systems with delay, J. Differential Equations 125(2) (1996), 441-489. DOI: 10.1006/jdeq.1996. 0037.

[35] J. Mawhin, The legacy of Pierre-François Verhulst and Vito Volterra in population dynamics, in: "The first 60 years of nonlinear analysis of Jean Mawhin", World Sci. Publ., River Edge, NJ, 2004, pp. 147-160. DOI: 10.1142/9789812702906_0012.

[36] R. M. MAY, Biological populations with nonoverlapping generations: stable points, stable cycles, and chaos, Science 186 (1974), 645-647. DOI: 10.1126/science.186.4164.645.

[37] R. M. MAY, Mathematical models in whaling and fisheries management, in: "Some mathematical questions in biology" (Proc. 14th Sympos., San Francisco, Calif., 1980), Lectures Math. Life Sci. 13, Amer. Math. Soc., Providence, R.I., 1980, pp. 1-64.

[38] R. M. MaY, Nonlinear phenomena in ecology and epidemiology, Ann. New York Acad. Sci.357(1) (1980), 267-281. DoI: 10.1111/j. 1749-6632.1980.tb29692.x. 
[39] R. M. May, G. R. Conway, M. P. Hassell, and T. R. E. Southwood, Time delays, density-dependence and singlespecies oscillations, J. Anim. Ecol. 43 (1974), 747-770.

[40] J. Maynard Smith, "Mathematical ideas in biology", Cambridge University Press, London, 1968.

[41] J. Maynard Smith, "Models in ecology", Cambridge University Press, Cambridge, 1974.

[42] J. D. Murray, "Mathematical biology", Second edition, Biomathematics 19, Springer-Verlag, Berlin, 1993. DOI: 10.1007/b98869.

[43] A. J. Nicholson, An outline of the dynamics of animal populations, Aust. J. Zool. 2(1) (1954), 9-65. DOI: 10.1071/Z09540009.

[44] J. F. Perez, C. P. Malta, And F. A. B. Coutinho, Qualitative analysis of oscillations in isolated populations of flies, J. Theoret. Biol. 71(4) (1978), 505-514. DOI : 10.1016/0022-5193(78)90321-1.

[45] G. Röst And J. Wu, Domain-decomposition method for the global dynamics of delay differential equations with unimodal feedback, Proc. R. Soc. Lond. Ser. A Math. Phys. Eng. Sci. 463(2086) (2007), 2655-2669. DOI: 10.1098/rspa.2007.1890.

[46] S. RuAn, Delay differential equations in single species dynamics, in: "Delay differential equations and applications", NATO Sci. Ser. II Math. Phys. Chem. 205, Springer, Dordrecht, 2006, pp. 477-517. DOI : $10.1007 / 1-4020-3647-7 \_11$.

[47] A. O. Shelton And M. Mangel, Fluctuations of fish populations and the magnifying effects of fishing, Proc. Natl. Acad. Sci. USA 108(17) (2011), 7075-7080. DOI: 10.1073/pnas.1100334108.

[48] H. Smith, "An introduction to delay differential equations with applications to the life sciences", Texts in Applied Mathematics 57, Springer, New York, 2011. DOI: 10.1007/978-1-4419-7646-8.

[49] C. E. TAYlor And R. R. Sokal, Oscillations in Housefly population sizes due to time lags, Ecology 57(5) (1976), 1060-1067. DOI: 10.2307/1941071.

[50] H. Teismann, R. Karsten, R. Hammond, J. Hardman, And J. FRANKLIN, On the possibility of counter-productive intervention: the population mean for blowflies models can be an increasing function of the death rate, J. Biol. Systems 17(4) (2009), 739-757. DOI: 10.1142/S0218339009003009.

[51] A. J. Terry and S. A. Gourley, Perverse consequences of infrequently culling a pest, Bull. Math. Biol. 72(7) (2010), 1666-1695. DOI: $10.1007 /$ s11538-009-9492-9. 
[52] H. R. Thieme, "Mathematics in population biology", Princeton Series in Theoretical and Computational Biology, Princeton University Press, Princeton, NJ, 2003.

[53] H.-O. Walther, The 2-dimensional attractor of $x^{\prime}(t)=-\mu x(t)+$ $f(x(t-1))$, Mem. Amer. Math. Soc. 113(544) (1995), 76 pp.

[54] E. M. Wright, A non-linear difference-differential equation, $J$. Reine Angew. Math. 194 (1955), 66-87.

[55] T. Yi AND X. Zou, Map dynamics versus dynamics of associated delay reaction-diffusion equations with a Neumann condition, Proc. R. Soc. Lond. Ser. A Math. Phys. Eng. Sci. 466(2122) (2010), 2955-2973. DOI : 10.1098/rspa.2009.0650.

Departamento de Matemática Aplicada II

Universidad de Vigo

36310 Vigo

Spain

E-mail address: eliz@dma.uvigo.es 